\title{
Décomposition des molécules polyatomiques dans un réacteur à plasma hors d'équilibre. I. $\mathbf{N H}_{3}$
}

\author{
D. Rapakoulias, M. Domange et J. Amouroux \\ E.N.S.C.P., Laboratoire de Génie Chimique, 11, rue Pierre-et-Marie Curie, 75231 Paris Cedex 05, France
}

(Reçu le 8 janvier 1981, révisé le 4 mars 1981, accepté le 10 mars 1981)

\begin{abstract}
Résuṃé. - Les plasmas à pression réduite sont des milieux hors d'équilibre complexes, au sein desquels coexistent plusieurs modes d'excitation. La réaction chimique globale a également lieu en plusieurs étapes élémentaires dont les réactifs et les produits sont en général excités.

Nous avons établi un modèle du réacteur en trois étapes (excitation, recombinaison à chaud, trempe) qui, appliqué à la décomposition de $\mathrm{NH}_{3}$, permet de dégager le processus $\left(\mathrm{NH}_{3}, \mathrm{X}\right) \rightarrow\left(\mathrm{NH}_{3}, \mathrm{~A}, \mathrm{~B}\right)^{*} \rightarrow\left(\mathrm{NH}, \mathrm{a}^{1} \Delta\right)$ comme principale réaction de la décomposition.

Nous utilisons également la théorie de la réactivité chimique en milieu hors d'équilibre pour interpréter l'évolution de la réactivité du plasma en fonction de la répartition énergétique sur les différentes espèces. Nous concluons que la décomposition est contrôlée par la température de vibration du plasma. Lorsque le système s'approche de l'équilibre le taux de décomposition diminue.
\end{abstract}

\begin{abstract}
Plasmas at reduced pressure are complex non-equilibrium systems, in which several excitation modes coexist. The global reaction also take place by means of many elementary process in which reactants and products are often excited states.

We establish a model of the reactor in three stages (excitation, hot recombinations, quench) which, when applied in $\mathrm{NH}_{3}$ decomposition, shows that the key reaction is $\left(\mathrm{NH}_{3}, \mathrm{X}\right) \rightarrow\left(\mathrm{NH}_{3}, \mathrm{~A}, \mathrm{~B}\right)^{*} \rightarrow\left(\mathrm{NH}, \mathrm{a}^{1} \Delta\right)$.

We also use the theory of chemical reactivity in non-equilibrium systems in order to interpret the variation of plasma's reactivity as a function of energy repartition over the excited species. We conclude that the decomposition process is controlled by the vibrational temperature of the plasma. As the system approaches equilibrium, the decomposition rate reduces.
\end{abstract}

1. Introduction. - L'étude de la réactivité chimique des milieux hors d'équilibre apparaît comme l'une des tendances de la chimie contemporaine. Aussi ces dernières années, de nombreuses équipes de recherche, notamment celle de J. C. Polanyi, en ont souligné l'importance afin d'expliquer les écarts observés par rapport à la réactivité des milieux en équilibre thermodynamique.

Ces faits essentiels reposent sur une répartition non homogène de l'énergie entre les différents degrés de liberté de la molécule. En conséquence les températures de vibration, de rotation et de translation des molécules sont différentes dans un système hors d'équilibre. Cette conclusion a comme corollaire que la réactivité chimique n'obéit plus aux lois prévues par la thermodynamique.

Cette théorie de la réactivité en milieu hors d'équilibre est basée sur les résultats expérimentaux obtenus dans des milieux relativement simples (les faisceaux moléculaires, notamment).
Les plasmas à pression réduite sont des milieux hors d'équilibre plus complexes, au sein desquels coexistent plusieurs modes d'excitation (vibrationnelle, rotationnelle, électronique, ionique, translationnelle). La réaction globale a lieu également en plusieurs étapes et dans chaque réaction élémentaire, les réactifs et les produits sont généralement excités.

Cette complexité explique le fait que les mécanismes réactionnels dans ce type de plasma sont encore mal connus. Il paraît donc intéressant d'utiliser la théorie de Polanyi pour expliquer la réactivité chimique du plasma.

Une première tentative dans ce sens nous a déjà permis d'interpréter la réactivité chimique lors de la fixation de l'azote dans un plasma hors d'équilibre $[3,12]$.

Nous allons dans ce travail étudier la décomposition de la molécule polyatomique $\mathrm{NH}_{3}$ dans un réacteur à plasma basse pression ( 5 à 50 mbar). Il s'agit d'un outil expérimental précieux puisque, selon nos travaux 
antérieurs $[11,12]$, la température de vibration et de rotation-translation des espèces dépend essentiellement d'une variable d'action : la pression. Dans ces conditions, l'analyse du niveau hors d'équilibre du système dépendra de la pression choisie pour l'expérience.

Trois raisons ont guidé le choix de la molécule $\mathrm{NH}_{3}$ :

- Premièrement, les voies de décomposition des molécules polyatomiques dans les plasmas hors d'équilibre sont peu étudiées.

- Deuxièmement, les intermédiaires réactionnels $\left(\mathrm{NH}, \mathrm{NH}_{2}\right)$ et les produits finaux $\left(\mathrm{N}_{2}, \mathrm{H}_{2}\right)$ sont parfaitement connus du point de vue spectral.

- Troisièmement, lors d'études antérieures nous avons étudié l'excitation de l'azote afin de réaliser sa fixation directe avec l'oxygène pour la synthèse de NO. La décomposition de $\mathrm{NH}_{3}$ permettra d'étudier le mécanisme inverse des études antérieures, à savoir les processus de formation de $\mathrm{N}_{2}$ par recombinaisons radicalaires.

Sur le plan appliqué cette étude est fort intéressante. Elle permet en effet l'obtention d'hydrogène ultra pur, hautement réactif lorsqu'il est à l'état atomique et parfaitement adapté à la régénération des catalyseurs.

Notre étude sera représentée en quatre parties : caractéristiques du montage expérimental et de l'outil analytique, résultats expérimentaux, mécanisme de dissociation, analyse des processus hors d'équilibre et en particulier rôle de l'excitation vibrationnelle.

2. Dispositif expérimental. - L'objectif de cet outil est de permettre l'établissement des corrélations entre les variables d'action et les variables macroscopiques, c'est-à-dire de relier l'état hors d'équilibre du réacteur et son taux de conversion.

L'appareillage est composë de trois parties (Fig. 1) :

- Le réacteur à plasma et les groupes d'alimentation en gaz et en énergie.

- L'analyse et le contrôle de la marche du réacteur (chromatographie, débits, pression, compteurs pour établir le bilan matière et énergétique).

- Le spectromètre d'émission, pour suivre in situ l'évolution des espèces excitées dans le réacteur en fonction des variables d'action et de l'état hors d'équilibre.

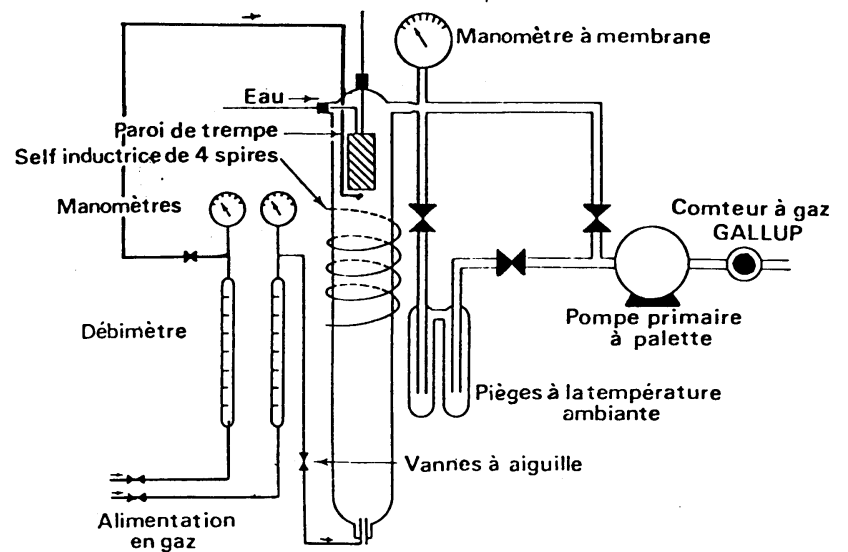

a)

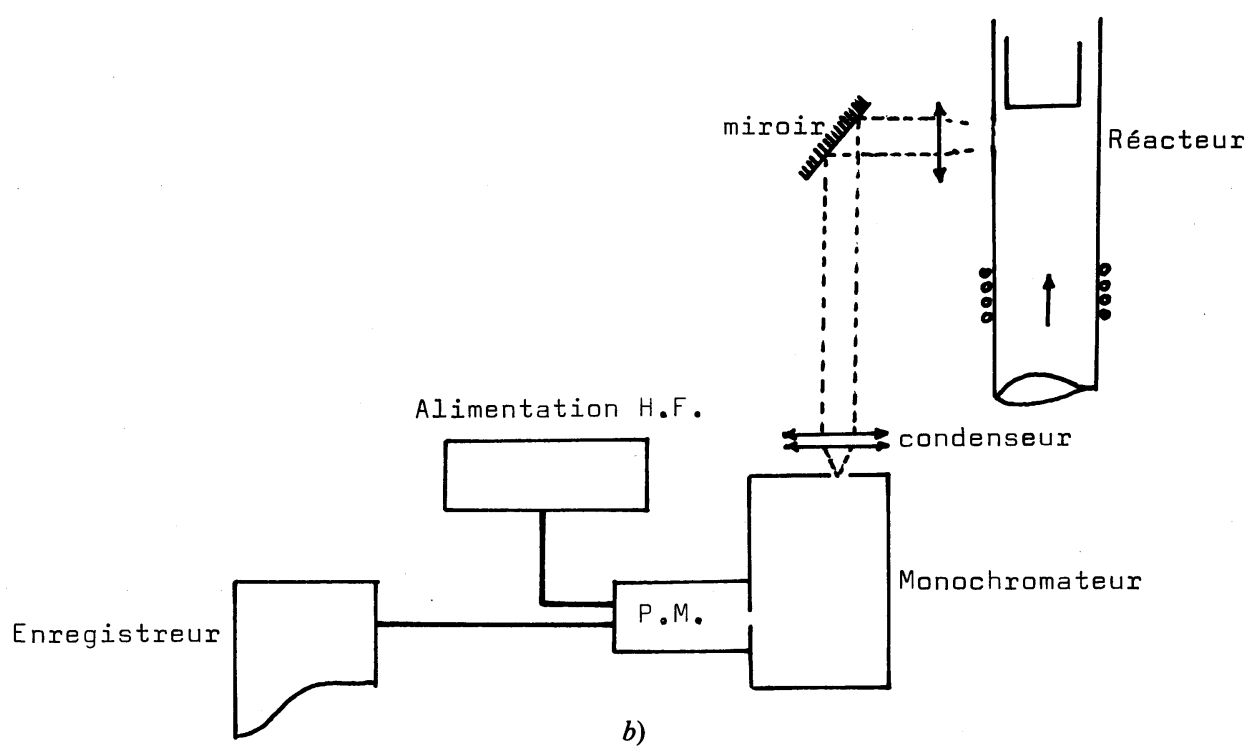

b)

Fig. 1. - a) Appareillage expérimental. b) Dispositif d'analyse spectroscopique.

[a) Experimental apparatus. b) Spectroscopic analysis apparatus.]

Les principales caractéristiques du réacteur sont les suivantes : il s'agit d'un réacteur tubulaire en verre de $0,10 \mathrm{~m}$ de diamètre et de $0,15 \mathrm{~m}$ de long, alimenté par de l'ammoniac sous une pression comprise entre 5 et 25 mbar. Le débit d'alimentation varie entre 0,5 et $2,0 \mathrm{l} / \mathrm{min}$., ce qui correspond à un temps de passage compris entre 0,3 et $1,2 \mathrm{~s}$. La puissance disponible aux bornes de l'inducteur est comprise entre 0,5 et $2,5 \mathrm{~kW}$.

Le contrôle du fonctionnement du réacteur est réalisé par une instrumentation située à l'entrée et à la sortie. Elle comprend les organes de mesure de 
débit, de pression et de volume, afin d'établir le bilan matière et énergétique.

La composition du mélange de sortie est déterminée par l'emploi d'un chromatographe en phase gazeuse comprenant une colonne de chromosorb 102 pour l'analyse de $\mathrm{NH}_{3}$ et une colonne de tamis moléculaire 13X montée en série pour l'analyse de $\mathrm{H}_{2}, \mathrm{~N}_{2}$ et $\mathrm{O}_{2}$.

Le rendement est donné par la relation

$$
x=\frac{\left(\mathrm{N}_{2}\right)}{\left(\mathrm{NH}_{3}\right)^{0}}=\frac{\left(\mathrm{NH}_{3}\right)^{0}-\left(\mathrm{NH}_{3}\right)}{\left(\mathrm{NH}_{3}\right)^{0}} .
$$

Les mesures spectroscopiques sont effectuées sur un monochromateur Jobin-Yvon HR2 muni d'un réseau de $2.400 \mathrm{tr} / \mathrm{mm}$ et d'un photomultiplicateur Hamamatsu R374. La plage de travail est de 2300 à $8000 \AA$ (Fig. 1b). Ce dispositif permet, par rotation du miroir, d'examiner toute la longueur du réacteur. Le spectre obtenu représente une mesure globale intégrée sur tout le rayon du réacteur.

La plupart des mesures sont réalisées au milieu du réacteur ou près de la paroi de trempe, selon la zone réactionnelle que l'on cherche à étudier (voir $§ 4.1$ ).

3. Résultats expérimentaux. - Nous avons cherché à établir de façon systématique les corrélations entre variables d'action du réacteur, son rendement et l'état énergétique des espèces excitées.

Pour la clarté de la présentation nous débuterons par l'examen des espèces excitées et de leur état énergétique dans le réacteur. Nous établirons dans un deuxième temps l'influence des variables d'action sur le rendement.

Le plasma de $\mathrm{NH}_{3}$ est parfaitement stable dans la région expérimentale étudiée. Sa couleur varie du blanc au jaune clair, selon les conditions expérimentales. L'émission lumineuse, beaucoup moins intense que dans les plasmas de $\mathrm{N}_{2}$ ou de $\mathrm{CH}_{4}$ est centrée dans l'axe du réacteur.

Le taux de décomposition de $\mathrm{NH}_{3}$ obtenu est important et peut dépasser $70 \%$. Les produits de la réaction sont $\mathrm{N}_{2}$ et $\mathrm{H}_{2}$. Nous n'avons pas détecté la présence de $\mathrm{N}_{2} \mathrm{H}_{4}$.

3.1 ANAlyse SPECTROSCOPIQUE. - 3.1.1 Aspect qualitatif du spectre d'émission. - La molécule de $\mathrm{NH}_{3}$ ne donne pas de spectre d'émission visible UV dans les décharges.

Parmi ses produits de décomposition nous avons détecté une très faible émission de bandes $\alpha$ de $\mathrm{NH}_{2}$. Les bandes de Schuster, caractéristiques de la formation d'hydrazine n'apparaissent pas.

La transition $\mathrm{A}^{3} \Pi \rightarrow \mathrm{X}^{3} \Sigma^{-}$du radical $\mathrm{NH}$ est très intense, tandis que l'émission de la transition $c^{1} \Pi \rightarrow \mathrm{a}^{1} \Delta$ est beaucoup plus faible.

L'intensité du deuxième positif de $\mathrm{N}_{2}$ est très forte. Par contre, nous n'avons pas détecté le premier système positif.

L'émission de l'ion $\mathrm{N}_{2}^{+}$(premier négatif) est faible. L'émission des atomes d'azote est très faible.
Enfin les raies $H(\alpha$ et $\beta$ ) sont présentes dans le spectre d'émission.

3.1.2 Aspect quantitatif. - Nous avons mesuré les températures de vibration et de rotation-translation des molécules en fonction des paramètres de travail. La première a été mesurée sur les bandes de la transition $\Delta v=2$ du deuxième positif de $\mathrm{N}_{2}$. La température de rotation-translation a été mesurée sur les raies de la bande $0-0$ de la transition $\mathrm{A}^{3} \Pi \rightarrow \mathrm{X}^{3} \Sigma^{-}$ de NH. Nous avons utilisé la méthode graphique de Boltzmann.

La température de rotation-translation du plasma (750 $\mathrm{K}$ en moyenne) est inférieure à la température de vibration (3 $500 \mathrm{~K}$ en moyenne). Un fort déséquilibre existe donc à l'intérieur du réacteur, comme c'était le cas pour le plasma de $\mathrm{N}_{2}, \mathrm{~N}_{2}+\mathrm{O}_{2}, \mathrm{~N}_{2}+\mathrm{CH}_{4}$ $[3,12]$.

L'augmentation de l'énergie induite élève légèrement toutes les températures. La modification de la pression fait baisser la température de vibration au profit de la température de rotation-translation (Fig. 2). Ce dernier résultat est dû à l'accélération du transfert $\mathrm{V} \rightarrow \mathrm{T}$ et $\mathrm{V} \rightarrow \mathrm{R}$ lorsque la densité du gaz augmente.

Enfin, la modification du débit ne provoque pas une variation importante des différentes températures.

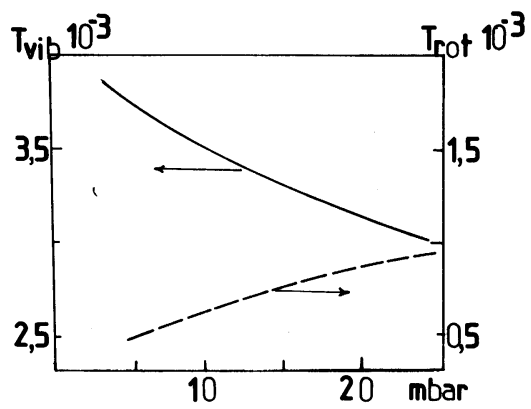

Fig. 2. - Variation des températures de vibration et de rotationtranslation avec la pression du réacteur

$$
\left(P I=0,8 \mathrm{~kW}, D_{\mathrm{NH}_{3}}=0,5 \mathrm{l} / \mathrm{min} \text {.) } .\right.
$$

[Variation of vibrational and translational temperatures as a function of pressure in the reactor.]

3.2 INFLUENCE DES PARAMÈTRES DE TRAVAIL. 3.2.1 Rôle de la pression. - Il s'agit du paramètreclef pour la modification de l'état d'équilibre du milieu chimique. Sa variation dans l'intervalle de 5 à 30 mbar a été effectuée à débit et puissance induite constants de $\left(D_{\mathrm{NH}_{3}}=0,5 \mathrm{l} / \mathrm{min}\right.$., $\left.P I=0,8 \mathrm{~kW}\right)$.

Le taux de décomposition de $\mathrm{NH}_{3}$ diminue quand la pression augmente (Fig. 3). L'interprétation du rôle de la pression sera effectuée à la fin de la quatrième partie.

3.2.2 Rôle de la puissance induite. - Ce paramètre a été étudié sous pression et débit constants ( $P=15 \mathrm{mbar}, D_{\mathrm{NH}_{3}}=0,5 \mathrm{l} / \mathrm{min}$.) dans l'intervalle de 0,5 à $3,0 \mathrm{~kW}$.

Une augmentation de la puissance induite provoque une augmentation de la population des espèces excitées 


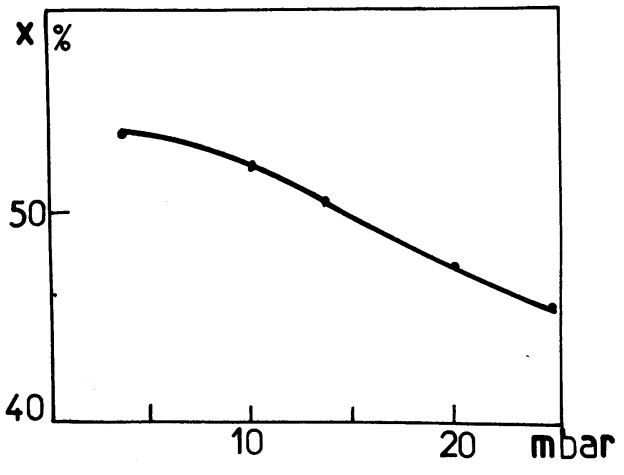

Fig. 3. - Variation du taux de décomposition avec la pression $\left(P I=0,8 \mathrm{~kW}, D_{\mathrm{NH}_{3}}=0,5 \mathrm{l} / \mathrm{min}\right.$.).

[Variation of the decomposition rate with pressure.]

et par conséquent du taux de décomposition (Fig. 4).

3.2.3 Rôle du débit. - La variation du débit de $\mathrm{NH}_{3}$ est étudiée sous pression et énergie induite constantes $(P=15 \mathrm{mbar}, P I=2,0 \mathrm{~kW})$.

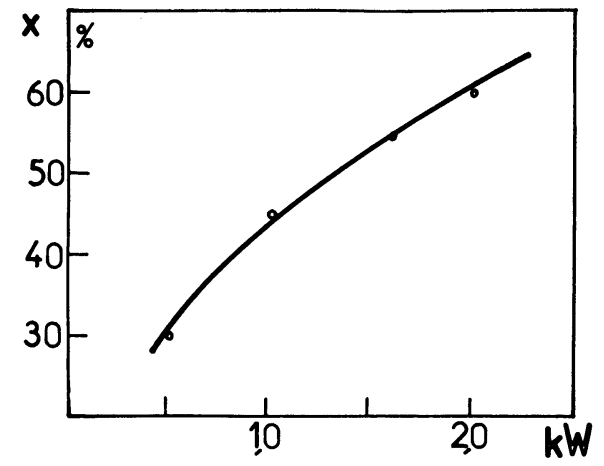

Fig. 4. - Variation du taux de décomposition de $\mathrm{NH}_{3}$ avec la puissance induite $\left(P=15 \mathrm{mbar}, D_{\mathrm{NH}_{3}}=0,5 \mathrm{l} / \mathrm{min}\right.$.).

[Variation of the decomposition rate of $\mathrm{NH}_{3}$ as a function of induced power.]

Les résultats, portés sur la figure 5 , montrent une diminution importante du taux de dissociation avec le débit. La quantité de $\mathrm{NH}_{3}$ décomposé (Fig. 6) se

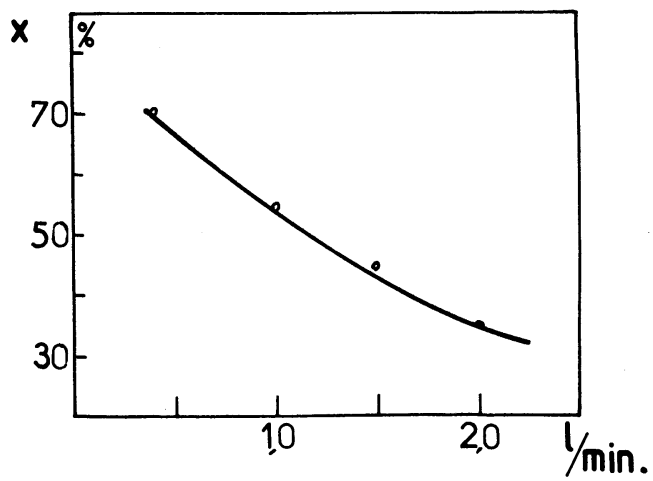

Fig. 5. - Variation du taux de décomposition de $\mathrm{NH}_{3}$ avec le débit à l'entrée du réacteur $(P=15 \mathrm{mbar}, P I=2,0 \mathrm{~kW})$.

[Variation of the rate of decomposition of $\mathrm{NH}_{3}$ as a function of gas flow rate at the entrance of the reactor.]

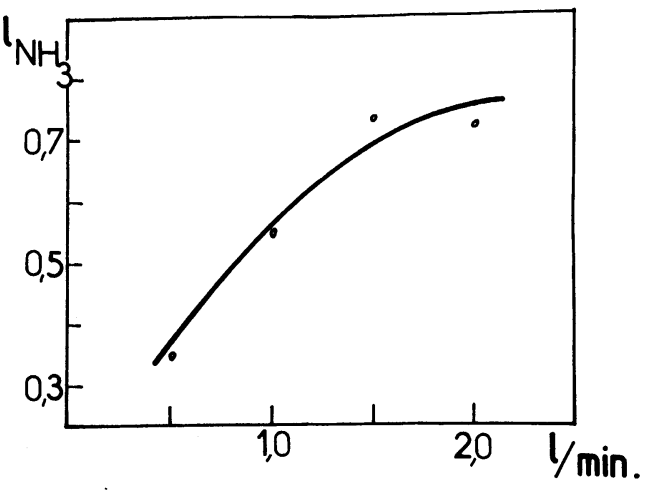

Fig. 6. - Variation de la quantité de $\mathrm{NH}_{3}$ décomposée avec le débit à l'entrée du réacteur $(P=15 \mathrm{mbar}, P I=2,0 \mathrm{~kW})$.

[Variation of the quantity of $\mathrm{NH}_{3}$ decomposed as a function of gas flow rate at the entrance of the reactor.]

stabilise aux environs de $0,7 \overline{1}$ dans les mêmes conditions expérimentales.

L'ensemble de ces résultats expérimentaux est résumé sur le tableau I où ils sont mis en corrélation avec l'étude de l'excitation interne du plasma. Les résultats expérimentaux obtenus antérieurement lors de la synthèse de $\mathrm{NO}$ et de $\mathrm{HCN}$ sont aussi indiqués sur ce tableau à titre de comparaison.

Tableau I.

\begin{tabular}{|c|c|c|c|c|c|}
\hline \multirow{3}{*}{$\begin{array}{l}\text { Variable } \\
\text { d'action }\end{array}$} & \multicolumn{2}{|c|}{$\begin{array}{l}\text { Spectro- } \\
\text { scopie }\end{array}$} & \multicolumn{3}{|c|}{ Réactivité chimique } \\
\hline & \multirow{2}{*}{$T_{\text {vib }}$} & \multirow{2}{*}{$T_{\text {rot }}$} & \multirow{2}{*}{$x_{\mathrm{NH}_{3}}$} & $\mathrm{~N}_{2}+\mathrm{O}_{2}$ & $\mathrm{CH}_{4}+\mathrm{N}_{2}$ \\
\hline & & & & $x_{\text {NO }}$ & $x_{\mathrm{HCN}}$ \\
\hline $\begin{array}{l}\text { Pression } \\
\text { Puissance } \\
\text { Débit entrée }\end{array}$ & $=$ & $=$ & 17 & $=$ & $=$ \\
\hline
\end{tabular}

4. Mécanisme de dissociation. - La décomposition de $\mathrm{NH}_{3}$ a fait l'objet de nombreux travaux de recherche appliquée, dans le but de fabriquer de l'hydrazine, combustible liquide pour les engins de propulsion [6]. Par contre, peu de travaux sont consacrés à une étude détaillée du mécanisme de décomposition, qui peut procéder par deux voies

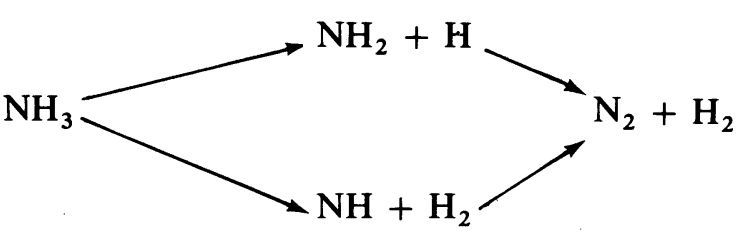

Carbaugh [7] suppose le radical $\mathrm{NH}_{2}$ comme l'intermédiaire de la décomposition. Cramarossa et al. [8] partent de l'autre hypothèse. L'équipe soviétique d'Eremin, lors de travaux destinés à la synthèse de $\mathrm{N}_{2} \mathrm{H}_{4}$ propose les deux voies à la fois $[9,10]$. Enfin, Capitelli et Molinari [4], proposent un mécanisme de 
dissociation thermique, par pompage de l'énergie du système sur les niveaux vibrationnels supérieurs fondamental de $\mathrm{NH}_{3}$.

4.1 ANALYSE DU RÉACTEUR A PLASMA HORS D’ÉQUILIBRE. - Notre approche du réacteur à plasma nous a conduit à scinder leur fonctionnement selon les 3 étapes suivantes :

a) L'excitation primaire (réacteur excitation) des réactifs où les collisions électron-molécule conduisent à l'excitation et éventuellement à la dissociation des réactifs. En ce qui concerne les liaisons chimiques, c'est une étape endothermique.

b) L'étape de recombinaison (ou réacteur chaud) où les espèces excitées produites à l'étape précédente interagissent entre elles pour la formation de nouvelles combinaisons entre atomes. C'est une étape quasiment adiabatique. Les nouvelles espèces produites sont généralement excitées.

c) Enfin l'étape de désexcitation et de recombinaison (ou réacteur de trempe) de toutes les espèces du plasma sur la paroi métallique refroidie. C'est une étape exothermique.

L'analyse spectroscopique longitudinale du réacteur permet d'établir la localisation approximative de ces trois étapes : trice ;

- l'excitation a lieu à l'intérieur de la self-induc-

- le réacteur chaud occupe la partie comprise entre l'inducteur et le voisinage de la paroi de trempe;

- le réacteur de trempe est situé au voisinage immédiat (de 1 à $2 \mathrm{~mm}$ ) de la paroi de trempe.

4.2 Réacteur d'excitation De $\mathrm{NH}_{3}$. - Dans le cas de $\mathrm{NH}_{3}$ cette première étape n'est pas sélective. En effet les collisions avec des électrons conduisent au peuplement de tous les états excités de $\mathrm{NH}_{3}$ (Fig. $7 a, b, c)$. Ces états sont dissociatifs ou se relaxent par collisions.

Compte tenu de la correspondance énergétique entre les différents états excités de $\mathrm{NH}_{3}$ et de ses fragments $\left(\mathrm{NH}_{2}\right.$ et $\left.\mathrm{NH}\right)$, la dissociation de $\mathrm{NH}_{3}$ est possible selon les réactions suivantes :

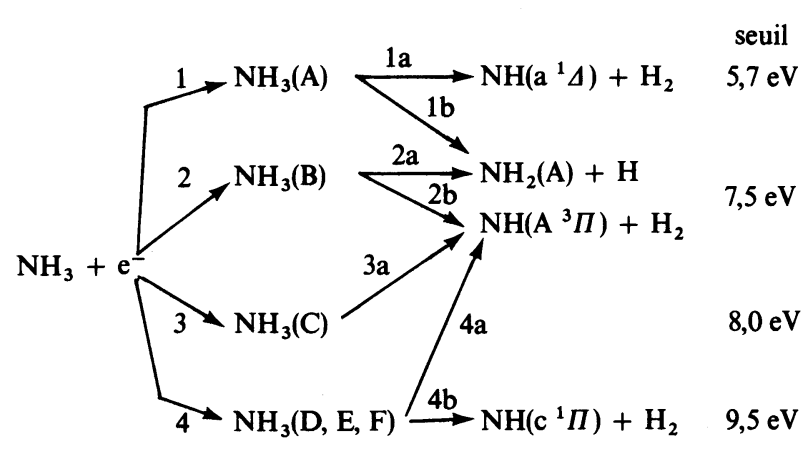

En partant de ce schéma de principe, les résultats de l'étude spectroscopique et les données de la bibliographie (tableau II) permettent de retenir trois réactions, dont la première est la plus importante voie de dissociation :

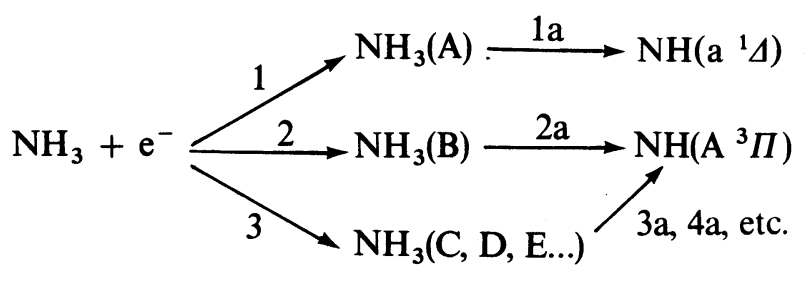

En effet, elle implique l'état de $\mathrm{NH}_{3}$ dont la section efficace d'excitation par collision avec les électrons est la plus importante et elle conduit à la formation d'un métastable de NH.

Les réactions 2 et 3 sont également importantes dans le réacteur compte tenu de la forte émission de la transition $\mathrm{A}^{3} \Pi \rightarrow \mathrm{X}^{3} \Sigma$. La faiblesse de l'émission $c^{1} \Pi \rightarrow \mathrm{a}^{1} \Delta$ de $\mathrm{NH}$ constatée lors de l'étude spectroscopique s'explique par le fait que la réaction $4 \mathrm{~b}$ comprend des états excités de $\mathrm{NH}_{3}$ de faible section efficace avec les électrons et qui plus est, correspondent à des niveaux d'énergie des électrons peu peuplées.

Remarque : Cette interprétation corrobore les résultats obtenus par Fukui et par Bubert concernant la dissociation de $\mathrm{NH}_{3}$ dans les canons à électrons (Fig. 8).

Tableau II.

Section efficace d'excitation de $\mathrm{NH}_{3}$ par collisions avec les électrons

$\begin{array}{cl}\text { Réaction } & \text { (Fig. 7) } \\ - & \text { importante } \\ \text { 1a } & \text { importante } \\ \text { 1b } & \text { faible } \\ \text { 2a } & \text { faible } \\ 2 \mathrm{~b} & \text { faible } \\ 3 \mathrm{a} & \text { importante } \\ \text { 4a } & \text { importante }\end{array}$

$$
\begin{aligned}
& \text { Population des } \\
& \text { électrons d'énergie } \\
& \text { correspondante } \\
& \text { dans la décharge } \\
& - \\
& \text { importante } \\
& \text { importante }
\end{aligned}
$$

faible faible

\section{Présence du} fragment correspondant dans le spectre d'émission

(métastable) très faible très faible importante importante importante faible

\section{Conclusion}

la plus importante peu probable peu probable probable probable probable peu probable 

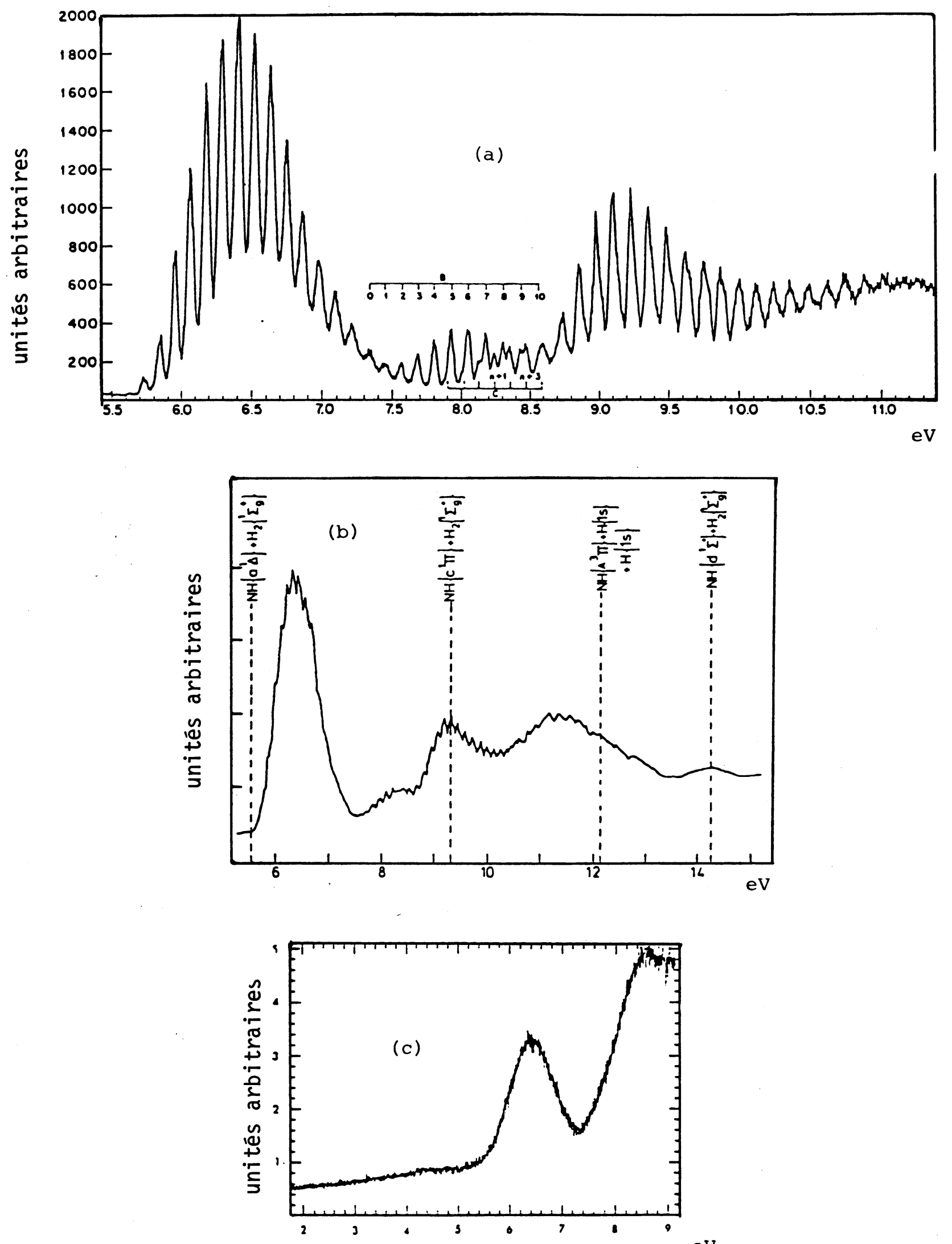

eV

Fig. 7. - Excitation de $\mathrm{NH}_{3}$ par collisions avec les électrons. La bande de 5,5 à 7,5 eV correspond à l'état $\tilde{\mathrm{A}}$ de $\mathrm{NH}_{3}$. Entre 7,5 et 8,6 eV les états $\widetilde{B}$ et $\widetilde{C}$ sont excités. Au-delà de $8,6 \mathrm{eV}$ apparaît l'excitation non résolue de deux ou plusieurs états. (a) d'après Harshbarger (1971); (b) d'après Johnson (1977); (c) idem.

[Electron impact excitation of $\mathrm{NH}_{3}$ molecule. The $5.5-7.5 \mathrm{eV}$ pic corresponds to the $\tilde{\mathrm{A}}$ state of $\mathrm{NH}_{3}$. In the 7.5-8.6 eV region $\tilde{\mathrm{B}}$ and $\tilde{\mathrm{C}}$ states are excited. Above $8.6 \mathrm{eV}$ several, non-resolved states are excited.] 

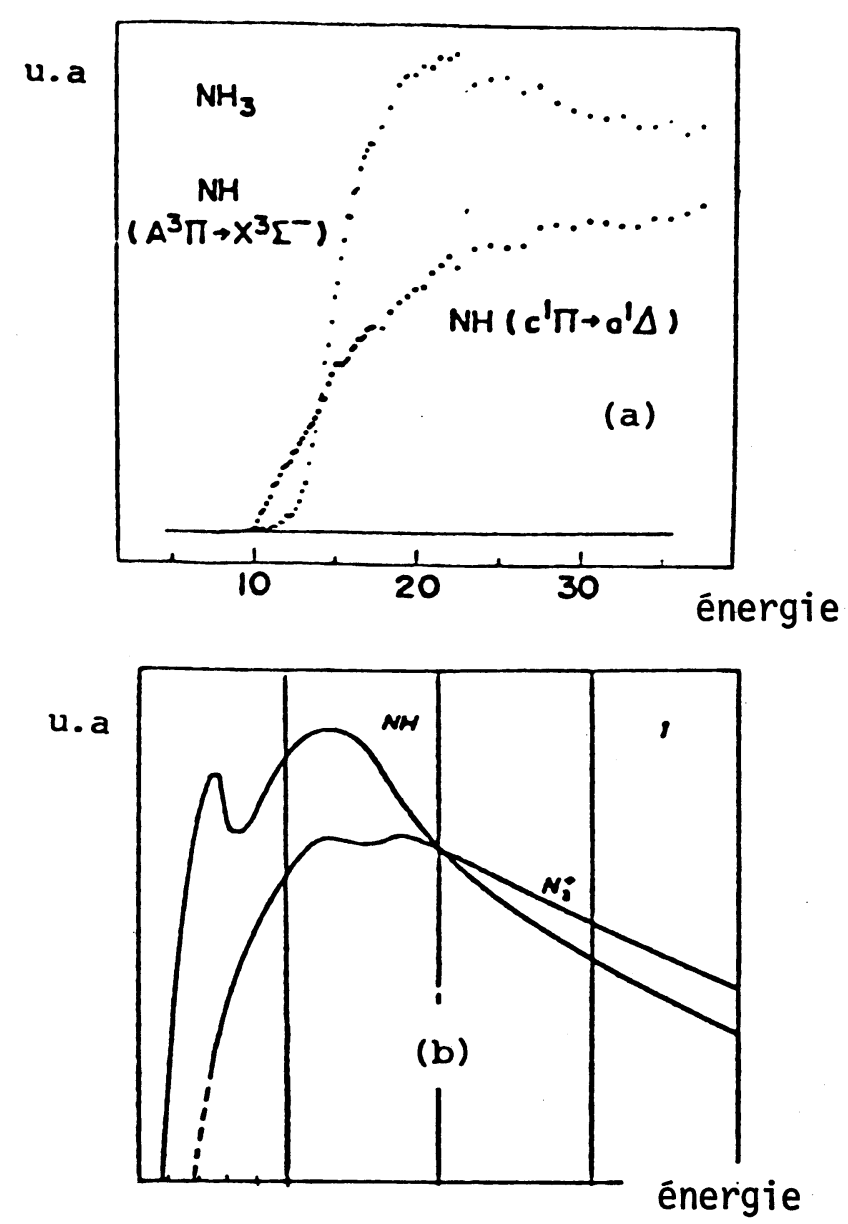

Fig. 8. - Section efficace de production des fragments de $\mathrm{NH}_{3}$ dans des canons à électrons. (a) d'après Fukui; (b) d'après Bubert.

[Cross section of $\mathrm{NH}_{3}$ fragments production in electron beam.]

4.3 RéacteUr CHAUd. - Les produits de l'excitation primaire peuvent donner lieu aux réactions suivantes :

$$
\begin{array}{ll}
\text { (5) } \mathrm{NH}+\mathrm{NH} \rightarrow \mathrm{N}_{2}^{*}+\mathrm{H}_{2}^{*} \\
\text { (6) } \mathrm{NH}+\mathrm{H} \rightarrow \mathrm{NH}_{2}^{*} \\
\text { (7) } \mathrm{NH}+\mathrm{NH}_{3} \rightarrow\left(\mathrm{N}_{2} \mathrm{H}_{4}\right) \\
& \mathrm{NH} \rightarrow \mathrm{N}\left({ }^{2} \mathrm{D}\right)+\mathrm{H}\left({ }^{2} \mathrm{~S}\right) \\
\text { (8) } & \mathrm{N}+\mathrm{N} \rightarrow \mathrm{N}_{2}^{*} \\
& \mathrm{H}+\mathrm{H} \rightarrow \mathrm{H}_{2}^{*} .
\end{array}
$$

Parmi ces réactions, la réaction (6) est peu probable car elle est exothermique et le radical $\mathrm{NH}_{2}$, formé à un état excité, serait détecté par la spectroscopie d'émission.

La formation d'hydrazine (réaction (7)) est peu probable dans le réacteur chaud. En effet, il s'agit d'une réaction exothermique et la molécule fragile, $\mathrm{N}_{2} \mathrm{H}_{4}$, formée à un état excité ne serait stable que si un troisième partenaire évacuait l'excès d'énergie (collision à trois corps). Ce type de processus a une faible probabilité dans un gaz à pression réduite. Nous expliquons ainsi l'absence dans le spectre d'émission de bandes de Schuster, caractéristiques de la formation de $\mathrm{N}_{2} \mathrm{H}_{4}$.

L'ensemble des réactions (8) est également exclu, car nous n'avons pas détecté d'émission d'atomes d'azote.

La réaction (5) semble ainsi être la réaction principale du réacteur chaud. Compte tenu du caractère quasi adiabatique de cette étape, les produits $\mathrm{N}_{2}$ et $\mathrm{H}_{2}$ sont formés à un état très excité, ce qui explique la forte émission des bandes de $\mathrm{N}_{2}$ et des raies $\mathrm{H}(\alpha, \beta)$.

\subsection{RÉACTEUR DE TREMPE}

$$
\begin{aligned}
& \text { (9) } \mathrm{NH}+\mathrm{NH} \rightarrow \mathrm{N}_{2}+\mathrm{H}_{2} \\
& \text { (10) } \mathrm{N}_{2}^{*} \rightarrow \mathrm{N}_{2} \\
& \text { (11) } \mathrm{H}_{2}^{*} \rightarrow \mathrm{H}_{2} \\
& 2 \mathrm{H} \rightarrow \mathrm{H}_{2} \\
& \text { (12) } \mathrm{NH}+\mathrm{H}_{2} \rightarrow \mathrm{NH}_{3} .
\end{aligned}
$$

La réaction de synthèse de l'hydrazine (7) n'a pas lieu dans ce réacteur, compte tenu de son absence dans les gaz de sortie. Ceci s'explique par le fait qu'elle est beaucoup moins exothermique $(20 \mathrm{kcal} / \mathrm{mol})$ que les réactions (9) $(160 \mathrm{kcal} / \mathrm{mol})$ et $(12)(80 \mathrm{kcal} / \mathrm{mol})\left({ }^{1}\right)$.

Nous arrivons ainsi au mécanisme global représenté sur la figure 9.

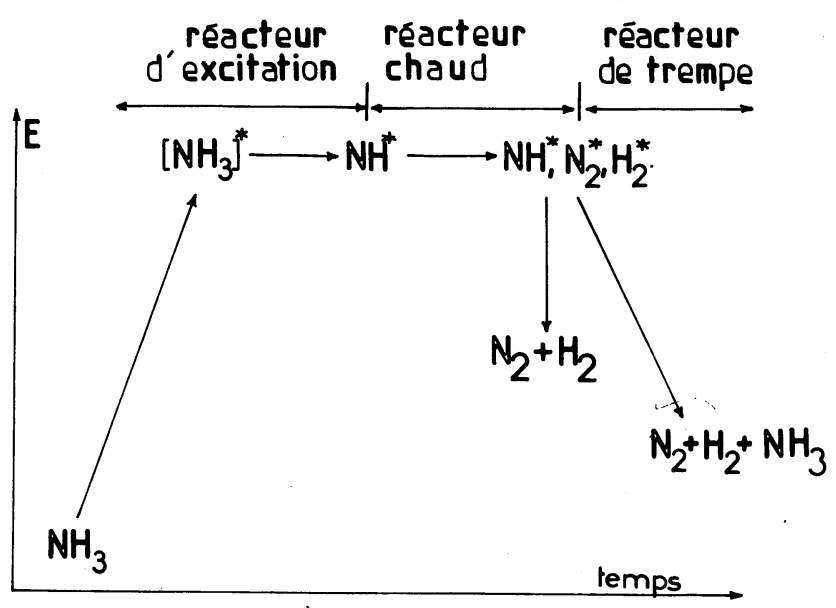

Fig. 9. - Diagramme énergétique représentant les différentes étapes de la dissociation de $\mathrm{NH}_{3}$ dans le réacteur.

[Energy diagram of the different stages of $\mathrm{NH}_{3}$ dissociation in the reactor.]

5. Rôle de la nature hors d'équilibre du plasma. 5.1 ETAPE-Clé De LA RÉACTION. RôLe DE L'EXCITATION VIBRATIONNELLE. - Lorsqu'une réaction a lieu dans un milieu hors d'équilibre, la répartition de l'énergie entre les différents modes d'excitation de la molécule influence fortement sa réactivité chimique. Pour les réactions en phase gazeuse, une forte excitation vibrationnelle favorise les processus endothermiques, tandis

$\left({ }^{1}\right)$ Ces chiffres n'ont qu'une valeur indicative. En effet, dans tous ces cas les réactifs et les produits peuvent être excités, tandis que les enthalpies indiquées concernent uniquement l'état fondamental. 
que les processus exothermiques sont favorisés par une énergie de translation élevée $[3,18]$.

Pour illustrer l'application de ces principes dans les plasmas hors d'équilibre nous pouvons rappeler les conclusions auxquelles nous sommes parvenus lors de travaux antérieurs. En effet la synthèse directe de $\mathrm{NO}$ (système $\mathrm{H}_{2}-\mathrm{O}_{2}$ ) et de $\mathrm{HCN}$ (système $\mathrm{N}_{2}-\mathrm{CH}_{4}$ ) en phase plasma nous a déjà permis de relier la réactivité chimique et l'excitation hors d'équilibre du plas-
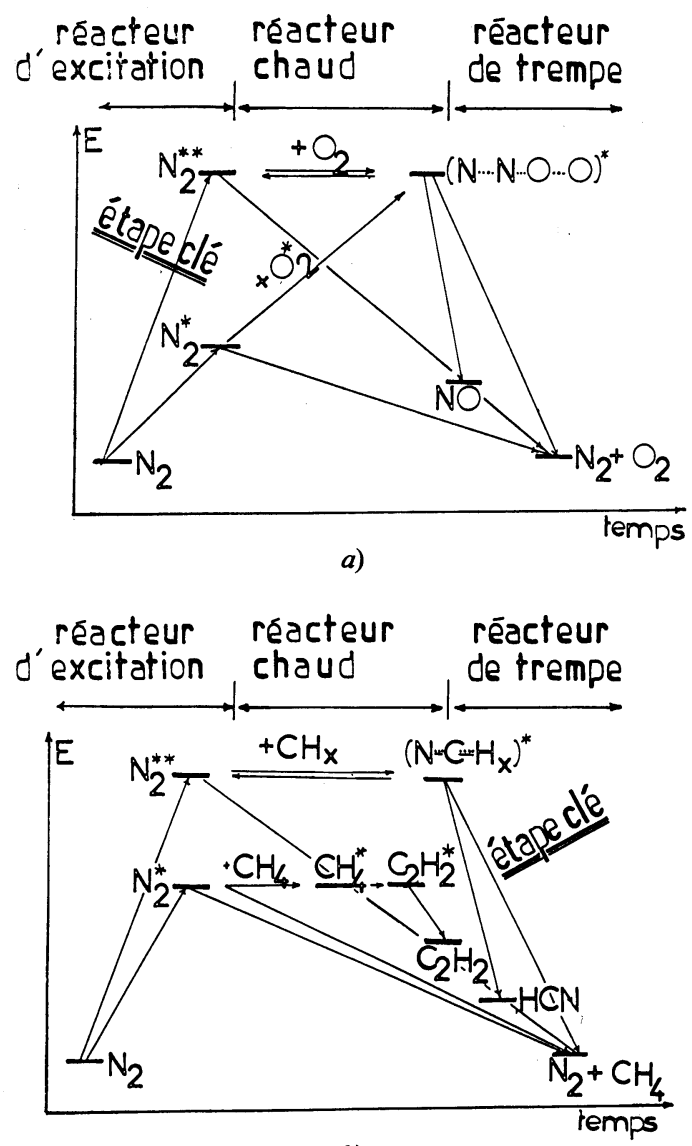

b)

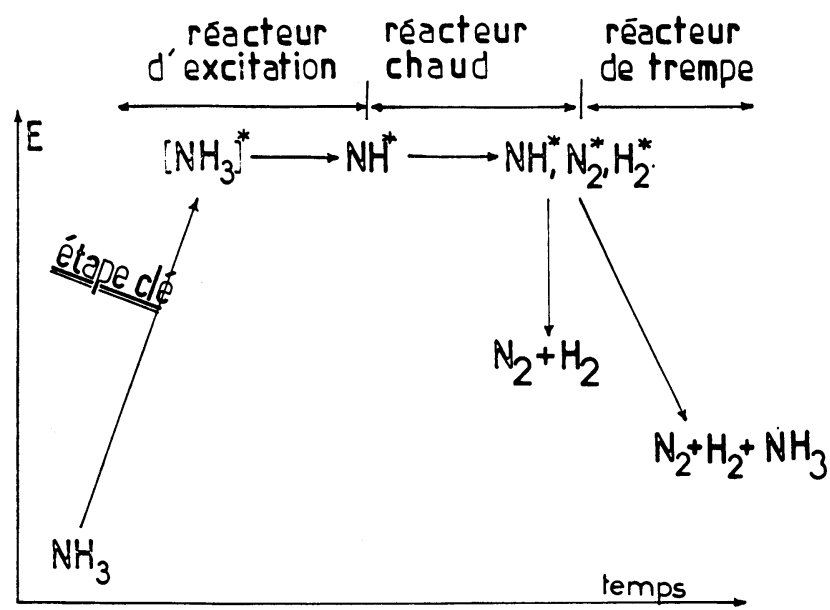

c)

Fig. 10. - Diagramme énergétique et étape-clé de la réaction globale : a) plasma $\mathrm{N}_{2}+\mathrm{O}_{2} ;$ b) plasma $\mathrm{N}_{2}+\mathrm{CH}_{4} ;$ c) plasma $\mathrm{NH}_{3}$.

[Energy diagram and control stage of the total reaction.] ma : l'accumulation d'énergie sur la vibration de $\mathrm{N}_{2}$ favorise la synthèse de NO, tandis que la formation de HCN est facilitée par une température de rotationtranslation élevée. En d'autres termes, l'étape-clé de la synthèse de NO est l'étape endothermique de dissociation du réactif $\mathrm{N}_{2}$ (réacteur d'excitation), alors que l'étape de la formation de $\mathrm{HCN}$ est l'étape endothermique de stabilisation du produit final (réacteur de trempe) (Fig. 10a,b).

Dans le cas de l'étude expérimentale de $\mathrm{NH}_{3}$, la relation entre le taux de conversion $x$ et l'écart de l'équilibre du plasma est illustrée sur la figure 11 sous la forme $x=f(\alpha)$, $\alpha$ étant un paramètre mesurant l'écart à l'équilibre défini par la relation

avec

$$
\alpha=\frac{T \mathrm{v}-T \mathrm{r}}{T \mathrm{~m}},
$$

$$
T \mathrm{~m}=\frac{T \mathrm{v}+T \mathrm{r}}{2} \quad(0<\alpha<2) .
$$

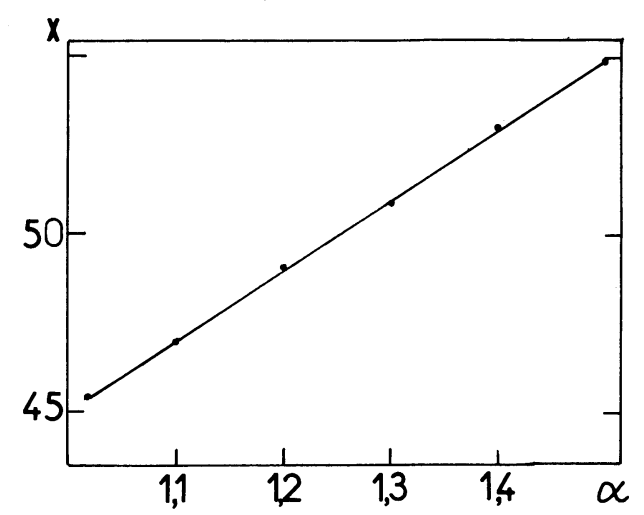

Fig. 11. - Variation du taux de décomposition en fonction de l'écart à l'équilibre (pression variable, $P I=0,8 \mathrm{~kW}, D_{\mathrm{NH}_{3}}=0,5 \mathrm{l}$ / $\min$.).

[Variation of decomposition rate as a function of deviation from equilibrium.]

Les mêmes résultats expérimentaux indiquent en fonction de la pression de l'excitation vibrationnelle varie comme le taux de décomposition de $\mathrm{NH}_{3}$ (Fig. 12), tandis que la température de rotationtranslation varie en sens inverse.

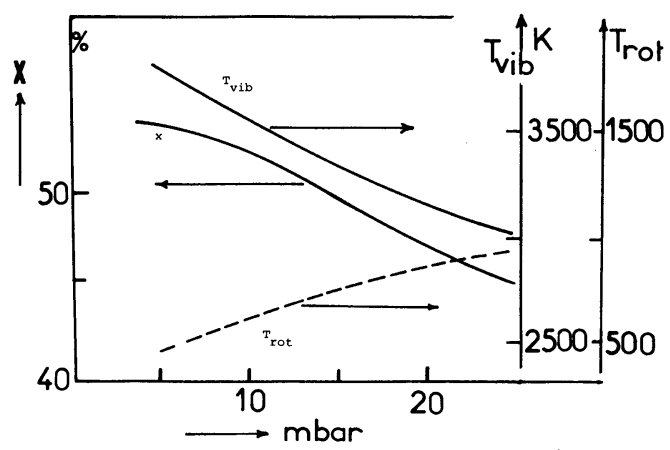

Fig. 12. - Variation simultanée du taux de décomposition et des températures de vibration et de rotation (pression variable, $P I=0,8 \mathrm{~kW}, D_{\mathrm{NH}_{3}}=0,5 \mathrm{l} / \mathrm{min}$.).

[Simultaneous variation of the decomposition rate and the vibrational and rotational temperature.] 
Cette conclusion signifie que l'étape déterminante de la réaction est une étape endothermique, compte tenu du mécanisme proposé (Fig. 9), elle ne peut être que l'étape d'excitation primaire de $\mathrm{NH}_{3}$ (Fig. 10c).

Ainsi la décomposition de $\mathrm{NH}_{3}$ peut être schématisée par les réactions consécutives suivantes :

$$
\mathrm{NH}_{3} \stackrel{k_{1}}{\longrightarrow} \mathrm{NH} \stackrel{k_{2}}{\longrightarrow} \mathrm{N}_{2}+\mathrm{H}_{2}
$$

pour lesquelles $k_{1}$ est bien inférieur à $k_{2}$.

Cette analyse est confirmée par l'étude spectroscopique de l'évolution du rapport $\mathrm{NH} / \mathrm{N}_{2}$ en 'fonction du taux de décomposition (Fig. 13). En effet, cette courbe est typique de l'évolution du rapport

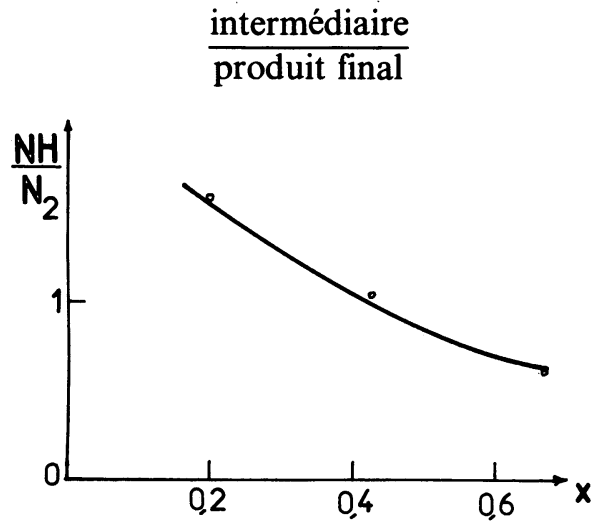

Fig. 13. - Variation du rapport $\mathrm{NH} / \mathrm{N}_{2}$ avec le taux de décomposition de $\mathrm{NH}_{3}$

[Variation of $\mathrm{NH} / \mathrm{N}_{2}$ rate as a function of $\mathrm{NH}_{3}$ decomposition rate.]

d'un modèle cinétique de type

$$
\mathrm{A} \stackrel{k_{1}}{\longrightarrow} \mathrm{B} \stackrel{k_{2}}{\longrightarrow} \mathrm{C}
$$

où $k_{1} \ll k_{2}$ (la simulation d'un tel modèle est indiquée sur la figure 14).

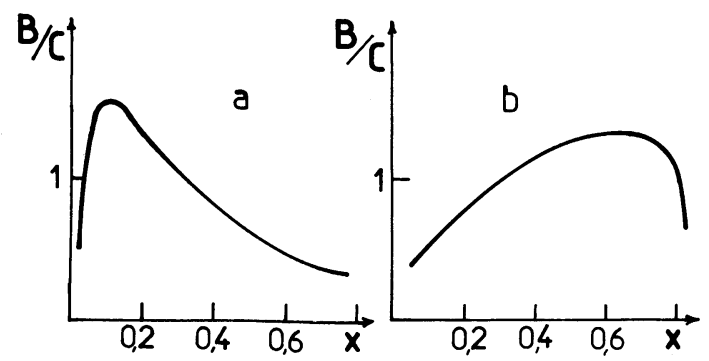

Fig. 14. - Simulation analogique de la variation du rapport $B / C$ avec le taux d'avancement de la réaction : $\mathrm{A} \stackrel{k_{1}}{\longrightarrow} \mathrm{B} \stackrel{k_{2}}{\longrightarrow} \mathrm{C}$. (a) $k_{1} / k_{2}=0,1 ;(b) k_{1} / k_{2}=10$.

[Analog simulation of the variation of $\mathrm{B} / \mathrm{C}$ rate as a function of reaction's progress.]
6. Conclusion. - L'analyse des réacteurs à plasma basse pression par la théorie de la réactivité chimique en milieu hors d'équilibre permet d'interpréter l'évolution de la réactivité en fonction de la répartition énergétique sur les différentes espèces du plasma.

La première conclusion importante précise que la décomposition de $\mathrm{NH}_{3}$ est contrôlée par la température de vibration du plasma. Lorsque le système se rapproche de l'équilibre, le taux de décomposition à l'intérieur du réacteur diminue.

En second, la réaction globale dans ce type de réacteur doit être analysée en trois étapes : excitation, recombinaison, trempe. L'étape-clé de la réaction globale est l'excitation primaire de $\mathrm{NH}_{3}$ suivie de sa rupture

$$
\mathrm{NH}_{3} \rightarrow\left(\mathrm{NH}_{3}\right)^{*} \rightarrow \mathrm{NH}+\mathrm{H} \quad\left(\text { ou } \mathrm{H}_{2}\right) .
$$

Le radical NH est le seul intermédiaire à longue durée de vie. Il se dissocie lors de l'étape de recombinaison selon la réaction

$$
2 \mathrm{NH} \rightarrow \mathrm{N}_{2}^{*}+\mathrm{H}_{2}^{*}
$$

et lors de la trempe

$$
2 \mathrm{NH} \rightarrow \mathrm{N}_{2}+\mathrm{H}_{2} .
$$

Ces résultats sont à rapprocher de ceux obtenus lors de la synthèse de NO (plasma $\mathrm{N}_{2}+\mathrm{O}_{2}$ ) et de $\mathrm{HCN}$ (plasma $\mathrm{N}_{2}+\mathrm{CH}_{4}$ ). Dans le cas de NO, l'étape-clé et la température de contrôle sont les mêmes que pour $\mathrm{NH}_{3}$. La synthèse de $\mathrm{HCN}$ par contre, est contrôlée par la température de translation et l'étape-clé est la trempe. Autrement dit, dans ce dernier cas le rendement augmente lorsqu'on se rapproche de l'équilibre.

Le caractère hors d'équilibre du plasma apparaît ainsi comme favorable à certaines synthèses et défavorable à d'autres. La définition de l'étape-clé permet aussi d'envisager des modifications du réacteur qui augmentent le rendement global.

Il faut toutefois souligner que toute cette analyse concerne uniquement la réaction en phase gazeuse, et ne tient pas compte des réactions possibles à la paroi du réacteur.

En effet, des réactions de surface - provoquées par la chimisorption de réactifs sur les parois du réacteur ou sur des surfaces métalliques introduites à cet effet dans le plasma - ont été détectées par notre équipe. Elles permettent d'envisager soit l'augmentation du rendement par réactions catalytiques hétérogènes, soit la modification permanente des surfaces métalliques (cémentation).

\section{Bibliographie}

[1] VEPREK, S., HERTZ, J., éd. 4th International Symposium on Plasma Chemistry, University of Zurich (1978).

[2] Fauchais, P., Baronnet, J.-M., Pure Appl. Chem. 52 (1980) 1721.

REVUE DE PHYSIQUe APPLIOUÚE. - - T. 16, No 6, JUIN 1981
[3] Rapakoulias, D., Thèse, Université Pierre \& Marie Curie (1979).

[4] Capitelli, M., Molinari, E., Topics in Current Chemistry 90 (Springer) 1980. 
[5] Polak, L. S., Slovetskyi, D. I., Int. J. Radiat. Phys. Chem. 8 (1976) 257.

[6] Voir références dans : Bibliography on Plasma Chemistry, IÜPAC, Subcommittee on Plasma Chemistry (1979).

[7] Carbaugh, D. C., Munno, F. J., Marchello, J. M., J. Chem. Phys. 17(12) (1967) 5211.

[8] Cramarossá, F., Coladrico, V., Agostino, R., Ferraro, G., MOLINARI, E., in : $3^{\mathrm{e}}$ symposium international de chimie des plasmas (édité par Fauchais, Limoges) 1976.

[9] Eremin, E. N., Syaduy, V. L., Russ. J. Phys. Chem. 49 (3) (1975) 316

[10] Syaduk, V. L., Eremin, E. N., Moscow Univ. Chem. Bull. 31 (1976) 529.

[11] Amouroux, J., Cavvadias, S., Rapakoulias, D., Revue Phys. Appl. 14 (1979) 969.

[12] Rapakoulias, D., Amouroux, J., Revue Phys. Appl. 14 (1979) 961.
[13] Harshbarger, N. R., J. Chem. Phys. 54(6) (1971) 2504

[14] Cvejanovic, D., Adams, A., King, G., J. Phys. B. 11(9) (1978) 1653.

[15] Johnson, E. K., Lipsky, S., J. Chem. Phys. 66(10) (1977) 4719.

[16] Bubert, H., Froben, F. W., J. Phys. Chem. 75(6) (1971) 769.

[17] Fukui, K., Fujita, I., Kuwata, U., J. Phys. Chem. 81(13) (1977) 1252.

[18] Polanyi, J. C., Acc. Chem. Res. 5 (1972) 161

[19] Amouroux, J., thèse, Université Paris VI (1971).

[20] Baddour, R., Timins, R., The application of plasmas to chemical processing (Pergamon Press) 1967.

[21] Blaustein, B. D. (éditeur), Chemical Reactions in Electrical Discharges (American Chemical Society) 1969.

[22] Travail de notre laboratoire, non publié.

[23] Cramarossa, F., Ferraro, G., J. Quant. Spectrosc. Radiat. Transfer 18 (1977) 471. 